Lelia Vitali

Karl-Franzens-Universität Graz

\title{
MIGRAZIONE ED IDENTITÀ: RIFLESSIONI TRADUTTIVE PARTENDO DAL CASO DI YOKO TAWADA
}

\section{Migration and identity: thoughts on translation based on Yoko Tawada's case}

\begin{abstract}
Taking as an example the emblematic case of the 'exophonic' writer Yoko Tawada, we will analyze some translation difficulties for the language pair German/Italian, which are due to linguistic differences and to topics related to the relationship between language and culture. In particular, we will examine two texts, originally written in German, for which an Italian translation has already been published. These works are Das Bad (1989) and Von der Muttersprache zur Sprachmutter (in Talisman, 1998) and focus thematically on personal and linguistic identity.
\end{abstract}

Keywords: exophony, literary translation, migration, identity, Tawada

\section{Riassunto}

Usando come esempio il caso emblematico di Yoko Tawada, autrice «exofonica», si analizzeranno alcune difficoltà traduttive per la coppia di lingue tedesco/italiano dovute a differenze linguistiche e a tematiche relative al binomio lingua/cultura. In particolare, si prenderanno in esame due testi originariamente in tedesco, già pubblicati in traduzione italiana: Das Bad (1989) e Von der Muttersprache zur Sprachmutter (in Talisman, 1998), che hanno al proprio centro il tema dell'identità personale e linguistica.

Parole chiave: exofonia, traduzione letteraria, migrazione, identità, Tawada

\section{Scrittori migranti}

Secondo una definizione di Armando Gnisci, la letteratura della migrazione è

prodotta da autori che scrivono in una lingua (nazionale) diversa da quella della fonte della propria provenienza, nella quale possono o no aver già scritto precedenti testi e/o continuare a scriverli, praticando o meno anche l'autotraduzione, in tutte e due le direzioni (Gnisci, 2003: 8). 
Si è già assistito all'intrigante fenomeno di scrittori che non si esprimono nella loro lingua madre (Kremnitz, 2004: 41). Già nel XX secolo l'irlandese Samuel Beckett tradusse Waiting for Godot nella sua lingua madre, l'inglese, dalla prima versione da lui scritta in francese dal titolo En attendant Godot. Un ulteriore esempio celebre è lo scrittore di origine polacca Józef Teodor Nałęcz Konrad Korzeniowski (Joseph Conrad), che scrisse Heart of Darkness e altri in inglese, sua terza lingua dopo il polacco e l'ucraino (Moretto, 2010: 72).

Esistono tuttavia anche fattori storici e socio-culturali che hanno creato veri e propri filoni di letteratura della migrazione espressa in una lingua non natia per gli scrittori.

Paesi quali Francia e Regno Unito hanno alle spalle un lungo passato coloniale, che ha avuto quale conseguenza un numero rilevante di autori migranti, ormai parte del canone della letteratura del Paese colonizzatore. Questa letteratura postcoloniale inizialmente comprendeva quegli autori provenienti dalle ex colonie che scrivevano nella lingua dei colonizzatori, ma con il tempo ha subito un importante allargamento, arrivando a comprendere anche gli autori originari di famiglie miste, emigrati o nati nell'ex Paese colonizzatore (Comberiati, 2010: 29-34). In ambito anglofono rientrano ad esempio in tale definizione Salman Rushdie, Hanif Kureishi e Chinua Achebe.

Paesi quali l'Italia e la Germania, che hanno avuto un passato coloniale modesto, hanno visto solo recentemente manifestazioni del fenomeno della letteratura della migrazione. A partire dagli anni Sessanta sono iniziati verso la Germania i primi flussi migratori di Gastarbeiter ingaggiati per dare un impulso alla ripresa economica nazionale, provenienti da Paesi quali Turchia, Grecia e Italia. Negli anni Novanta è poi stato il turno dell'Italia di diventare meta di migrazioni, grazie alla sua posizione strategica al centro del Mediterraneo (Moretto, 2010: 72).

Esempi di scrittori in tedesco sono Gino Carmine Chietino e Franco Biondi, entrambi di origine italiana, vincitori nel 1987 del premio Adelbert von Chamisso, riservato ad autori che non provengono da una nazione di lingua tedesca. Tra gli scrittori che usano l'italiano ricordiamo Amara Lakhous, il cui Scontro di civiltà per un ascensore a Piazza Vittorio (2006) è un'autotraduzione di una prima versione redatta in arabo (Lusetti, 2017: 109).

Esistono tuttavia altre possibili definizioni per autori che non usano la propria madrelingua per una scrittura creativa, che implicano un diverso approccio allo studio di tale opere, come si vedrà nel paragrafo seguente. 


\section{Scrittori 'exofonici'}

Si definiscono 'exofonici' gli scrittori che hanno scelto di produrre opere in una lingua che non è quella natia (Moro, 2018a: 9). Molti di questi autori presentano una produzione letteraria bilingue, con opere in entrambe le lingue, testi plurilingui o eterolingui, o autotraduzioni, in cui queste si hanno quando l'autore funge da traduttore e traduce autonomamente una propria opera (Lamping, 1992: 213).

L'adozione di tale termine, dall'inglese exophony/exophonic, serve a creare una distinzione fondamentale tra la produzione di scrittori di origine bilingue o mista e scrittori non madrelingua che hanno scelto attivamente di scrivere in un'altra lingua per questioni di stile o di creatività (Wright, 2008: 26). Aiuta inoltre a distaccarsi dalle tematiche trattate e a concentrarsi sulle caratteristiche stilistiche innovative di questi testi. Si tratta di una "zona grigia”, a metà tra la letteratura del Paese di origine e quella del Paese ospitante (Wright, 2008: 27).

Il termine sta prendendo sempre più piede anche perché incoraggia l'accettazione dell'uso in letteratura di una lingua straniera, o di più lingue, in quanto fenomeno naturale e destinato a prosperare come conseguenza della globalizzazione. Rappresenta inoltre un mutamento nell'approccio all'analisi di testi scritti da non madrelingua, incentrandosi sulla relazione tra forma e significato in letteratura (Wright, 2008: 40).

Exophonie (2003) è anche il titolo di una collezione di saggi di tema linguistico di Yoko Tawada, autrice che verrà trattata dettagliatamente nel prossimo paragrafo.

\section{Case study: Yoko Tawada}

All'interno del paradigma appena descritto degli autori cosiddetti 'exofonici', la scrittrice Yoko Tawada rappresenta un caso emblematico. Nata a Tokyo nel 1960, studia lingua e letteratura russa presso la Waseda University e, dopo la laurea, intraprende un viaggio con la ferrovia transiberiana, che la porterà per la prima volta in Europa. Nel 1982 decide quindi di trasferirsi ad Amburgo pur non sapendo ancora il tedesco e nel 1990 inizia a studiare Letteratura Tedesca, per conseguire successivamente il dottorato presso l'Università di Zurigo (Moro, 2018b: 98).

La sua produzione scritta è ad oggi molto variegata e corposa, comprendente decine di romanzi, racconti brevi, saggi letterari, poesie e pièce teatrali, sia in tedesco sia in giapponese. La sua prima opera pubblicata in Germania risale al 1986 e in Giappone al 1992 (Kloepfer, Matsunaga, 2000: 2). Ha ricevuto numerosi premi e riconoscimenti letterari, sia in Giappone sia in Germania, 
inclusi il premio Akutagawa (1992), il premio Adelbert von Chamisso (1996), il premio Tanizaki (2003), la medaglia Goethe (2005) e il premio Kleist (2016).

La figura di Tawada è quella di una scrittrice in viaggio, definibile 'un soggetto nomade', pur tenendo conto della differenza dovuta a certi privilegi socio-economici rispetto a scrittori della letteratura della migrazione (Braidotti, 2010). La sua condizione di «straniera», a metà tra due sfere linguisticoculturali molto distanti tra loro, la rende una scrittrice capace di una prosa ricca di suggestioni culturali e di profonde riflessioni linguistiche (Tawada, 2004: 40-41). Rimette in discussione le nozioni di identità, cultura, appartenenza nazionale e linguistica, focalizzandosi stilisticamente sulle interazioni tra il tedesco e il giapponese, che nelle sue opere danno vita a sperimentazioni linguistiche e testuali inedite di grande creatività (Capano, 2008).

Protagonisti delle sue opere sono quasi sempre figure migranti, attraverso il cui sguardo vengono demoliti i concetti di individualità e identità. Al centro sono poste spesso figure di interpreti o traduttori, che si scontrano a volte con l'incomunicabilità, il fraintendimento, l'ambiguità e il non sentirsi a casa in nessuna delle due culture che conoscono; o che a volte abbracciano la ricchezza di questa dualità, fruendo della libertà di essere al di fuori da strutture preconcette (Moro, 2018a: 25). Come l'autrice stessa, le figure nei suoi racconti sono quindi soggetti nomadi, in viaggio, ma per cui la migrazione non è vista più come una costrizione, come spesso accade nella letteratura della migrazione, ma più come un percorso voluto alla ricerca della propria identità.

Si può affermare che la figura di Yoko Tawada sia uscita dalla letteratura di nicchia per riscuotere sempre più interesse a livello internazionale presso il grande pubblico, soprattutto grazie a un romanzo scritto originariamente in giapponese e poi autotradotto dall'autrice in tedesco: si tratta di Yuki no renshuusei (L'apprendista di neve [trad. della sottoscritta], 2011)/Etüden im Schnee (2013), tradotto nel 2016 in inglese da Susan Bernofsky con il titolo Memoirs of a Polar Bear e a novembre 2017 in italiano da Alessandra Iadicicco con il titolo Memorie di un'orsa polare, edito da Guanda. Tale romanzo, che tratta la storia di tre generazioni di orsi migranti, è stato accolto con entusiasmo dalla critica internazionale e la versione inglese ha vinto la prima edizione del Warwick Prize for Women in Translation (2017).

\section{La traduzione in italiano delle opere di Yoko Tawada}

Negli ultimi anni gli studi sulla letteratura postcoloniale e della migrazione, nonché sulle loro peculiarità linguistiche e contenutistiche, si sono moltiplicati. È necessario tenere in considerazione che esiste una ricca produzione di autori in una lingua non natia, ma l'interesse del presente contributo si focalizza in particolare su come la scrittura dell'autrice in considerazione venga arricchita 
della sperimentazione linguistica in ambito letterario, come conseguenza dell'interazione tra due lingue diverse, spesso in modo inedito, che sfocia in testi con caratteristiche innovative.

Esistono pochissime traduzioni italiane delle opere di Yoko Tawada, per le difficoltà rappresentate sia dalla prosa sperimentale dell'autrice, in cui si fondono elementi di tedesco e di giapponese, sia per lo stile testuale peculiare. Tuttavia questa situazione sta mutando: come già detto, in ambito accademico l'interesse verso la letteratura della migrazione e l'eterolinguismo sta crescendo in maniera esponenziale, sia dal punto di vista antropologico, sia da quello delle implicazioni che il plurilinguismo porta con sé sotto l'aspetto traduttivo. Nell'odierno mondo globalizzato il grande pubblico ama ormai confrontarsi con altre lingue e culture, come dimostra il successo editoriale di Memoirs of a Polar Bear (2016).

Fino a due anni fa l'unica opera tradotta in italiano di Yoko Tawada era Das Bad (1989), scritto originariamente in giapponese e tradotto in tedesco da Peter Pörtner, pubblicato in Italia con il titolo Il Bagno (2003) in traduzione di Laura Aversa e Lucia Perrone Capano, che tratta il tema della metamorfosi femminile in chiave postmoderna.

Nel giro di appena sei mesi le opere di Yoko Tawada tradotte in italiano sono triplicate, con la traduzione dal tedesco di Alessandra Iadicicco di Memorie di un'orsa polare (2017, novembre) e la prima traduzione dal giapponese di Daniela Moro di Persona (2018, marzo). Questa seconda opera è un racconto breve pubblicato per la prima volta nel 1992 con il titolo giapponese Perusona, in un volume insieme all'opera vincitrice del Premio Akutagawa Inu muro iri (Il cane sposo) ed è una riflessione sulla relazione tra soggettività individuale e identità nazionale e culturale (Moro, 2018a: 10).

La novità introdotta dall'analisi di Yoko Tawada in chiave traduttiva è il fatto che l'eterolinguismo e la traduzione diventano i temi stessi delle opere dell'autrice, che ne fa l'oggetto di riflessione principale, dal punto di vista linguistico e culturale, e sono alla base di tutta la sua produzione letteraria.

Come affermano alcuni critici, senza questo connubio tra giapponese e tedesco la produzione dell'autrice non sarebbe esistita, o sarebbe stata declinata sotto un profilo totalmente diverso. (Sgambati, 2014). Tawada teorizza la presenza di "fessure" o "buchi" nel tessuto linguistico (Lücke in tedesco, sama, in giapponese), all'interno dei quali si inserisce la produttività linguistica (Moro, 2018a: 25).

Per quanto riguarda la traduzione, l'autrice demolisce la dualità tra «originale»e «traduzione», affermando che anche la prima stesura di un testo, il cosiddetto «originale», altro non è che una traduzione in scrittura di un'idea e che quindi, traducendolo poi da una lingua all'altra, ciò a cui bisogna rimanere fedeli non è il testo, ma l'anima dello stesso (Kloepfer, 1998: 14-15). 


\section{Analisi traduttive}

Si prenderanno ora in considerazione brani tratti da due opere di Yoko Tawada già pubblicate in traduzione italiana: Das Bad (1989), con il titolo italiano Il Bagno (2003), e Von der Muttersprache zur Sprachmutter (in Talisman, 1998), pubblicata in italiano sulla rivista Lettera Internazionale con il titolo Dalla Linguamadre alla Madrelingua (2012).

Come accennato in precedenza, le strutture grammaticali e sintattiche della lingua svolgono un ruolo di primo piano nella poetica dell'autrice, in un contesto «exofonico» (Agnese, Ivanovic, Vlasta, 2015: 107-125). Questa autoriflessione linguistica rende difficile la traduzione in un'altra lingua senza perdita di significato, ma lo scopo di Tawada è appunto quello di rendere poeticamente produttive queste differenze $\mathrm{o}$ «fessure» tra le lingue. Secondo Benjamin Walter (1972) la traduzione di un'opera letteraria poi altro non è che mera forma, senza scopi comunicativi verso il lettore. Infatti, neanche l'originale di un testo poetico è destinato ad un pubblico preciso di lettori, ma anzi la forza di alcune opere risiede proprio nella loro traducibilità o intraducibilità, nel rapporto intimo e naturale tra originale e traduzione.

Si veda ora quali strategie sono state adottate per far fronte alle varie difficoltà traduttive.

\subsection{Il bagno}

Il romanzo breve dallo stile onirico narra la storia di un'interprete giapponese in Germania che, nello svolgimento del proprio lavoro, ha una crisi di identità. Come in sogno, l'incontro con il partner tedesco prima, con la propria madre e lo spettro di una donna morta poi, portano a demolire pezzo dopo pezzo l'identità dell'io narrante, fino all'annullamento totale di qualsiasi individualità. Si prenda ora in esame un primo brano:

Dass ich auf Seezunge bestehe, liegt aber nicht nur an ihrem Geschmack. Es liegt an der "Zunge" der Seezunge. Wenn ich sie esse, habe ich das Gefühl, dass eine andere Zunge für mich weiterspricht, wenn ich einmal um Worte verlegen bin (Tawada, 1989: 41).

In traduzione italiana di Laura Aversa e Lucia Perrone Capano:

Il fatto che insista per avere una sogliola non dipende solo dal sapore, ma dalla "lingua" della sogliola. Quando la mangio ho come la sensazione che, nel momento in cui mi mancano le parole, un'altra lingua parli per me (Tawada, 2003: $35-36)$. 
Questo passaggio, in tedesco, presenta un gioco di parole intraducibile, tra Zunge, 'lingua', e Seezunge, 'sogliola', letteralmente 'lingua di mare'. Qui l'io narrante sta dicendo che ha l'impressione che mangiare una sogliola la possa aiutare nel momento in cui le mancano le parole per tradurre, essendo la sogliola appunto una 'lingua di mare'. Questa scomposizione della lingua nei suoi elementi primari, in questo caso di un Kompositum nei due sostantivi che lo formano, porta a riflessioni non scontate ed è parte integrante della poetica di Tawada. Purtroppo, in questo caso una perdita di significato in traduzione è stato inevitabile.

Si consideri ora un secondo brano:

Weil ich keine Zunge habe, kann ich nicht dolmetschen, kann ich, was jene Frau sagt, nicht ins Leichtverständliche übersetzen. Weil ich die Buchstaben vergessen habe, bin ich auch keine Typistin mehr. [...] Erst recht bin ich kein Fotomodell, denn ich bin auf Fotos gar nicht zu sehen.

Ich bin ein transparenter Sarg (Tawada, 1989: 163-165).

In traduzione italiana:

Poiché non ho la lingua, non posso fare l'interprete, non posso tradurre in qualcosa di facilmente comprensibile ciò che dice quella donna. Poiché ho dimenticato le lettere dell'alfabeto, non sono più neanche una dattilografa. [...] Una fotomodella poi non lo sono assolutamente, in quanto sulle foto non mi si vede.

Sono una bara trasparente (Tawada, 2003: 85).

Questo brano corrisponde alla conclusione del racconto. Non si riscontrano qui particolari difficoltà linguistiche nella traduzione, ma è un passaggio emblematico nella scomposizione dell'identità dell'io narrante femminile sotto lo sguardo e le aspettative altrui. La protagonista non riesce infatti a soddisfare più le aspettative di chi la circonda e rinuncia infine alla propria identità, sia personale che linguistica.

\subsection{Dalla Linguamadre alla Madrelingua}

Questo racconto breve, contenuto nella raccolta Talisman (1998), tratta della poetica dell'autrice relativa all'impatto personale e fisico che l'apprendere una nuova lingua porta con sé. Tawada afferma che si tratta dell'immersione in un nuovo mondo, in una nuova realtà, dove neanche gli oggetti sono più gli stessi, ma acquisiscono una nuova identità. Si veda a tal proposito il passaggio seguente:

Ein deutscher Bleistift unterscheidet sich kaum von einem japanischen. Er hieß aber nicht mehr "Enpitsu", sondern "Bleistift". Das Wort "Bleistift" machte mir den Eindruck, als hätte ich es jetzt mit einem neuen Gegenstand zu tun. Ich hatte 
ein leichtes Schamgefühl, wenn ich ihn mit dem neuen Namen bezeichnen musste (Tawada, 1989: 9).

Tradotto in italiano da Gabriella Sgambati come segue:

Una matita tedesca si differenziava di poco da una matita giapponese. Solo, non si chiamava più enpitsu, ma lapis. La parola lapis mi dava l'impressione di avere a che fare con un nuovo oggetto. Provavo un vago imbarazzo, quando dovevo definirlo con il nuovo nome (Sgambati, 2012: 45).

Questo brano esemplifica la personificazione degli oggetti da parte dell'io narrante, in quello che può essere definito un rapporto soggettivo con oggetti inanimati, in una prospettiva che Tawada definisce «animismo» tedesco.

Si prenda ora in esame un secondo brano:

Es gab auch ein weibliches Wesen auf dem Schreibtisch: eine Schreibmaschine. [...] Wenn ich mich vor sie hinsetzte, hatte ich das Gefühl, dass sie mir eine Sprache anbot. Ihr Angebot änderte zwar nichts an der Tatsache, dass Deutsch nicht meine Muttersprache ist, aber dafür bekam ich eine neue Sprachmutter. Diese neue Maschine, die mir eine Sprache schenkte, nannte ich Sprachmutter (Tawada, 1989: 13).

Ora la traduzione italiana:

C'era anche un essere femminile sulla scrivania: una macchina da scrivere. [...] Se mi sedevo davanti a lei, avevo la sensazione che mi offrisse una lingua. L'offerta non cambiava il fatto che il tedesco non fosse la mia Linguamadre, ma grazie a ciò ho ricevuto una nuova Madrelingua. Questa macchina femminile mi ha donato una lingua e la chiamavo Madrelingua (Sgambati, 2012: 46).

All'oggetto di genere maschile, il "lapis", viene contrapposto un essere femminile, a cui viene attribuita l'identità di "madre", che adotta l'io narrante e le regala una seconda infanzia all'interno della realtà dominata e definita da una seconda lingua. Dal punto di vista linguistico, la traduzione di questo testo presenta difficoltà di concordanza del genere dei sostantivi tra le due lingue: mentre infatti Bleistift è maschile, in italiano il traducente 'matita' sarebbe femminile. Se fosse stato usato quest'ultimo traducente si sarebbe però poi persa questa differenza successiva, in cui in mezzo a tanti oggetti maschili, che confondono e imbarazzano l'io narrante, vi è finalmente un essere femminile, una "madre", che offre accesso al mondo linguistico. Per evitare questa perdita di un importante passaggio, Sgambati ha giustamente risolto la difficoltà preferendo il traducente 'lapis', meno comune e forse con connotazione regionale, ma di genere maschile. 


\section{Conclusione}

Yoko Tawada si inserisce nel filone degli scrittori migranti ed 'exofonici', ma, come si è visto, le sue opere offrono spunti di riflessione interessanti per ulteriori studi nel campo dell'identità linguistica e della traduzione. Si è analizzato come l'approccio traduttivo dei suoi testi in una lingua terza, rispetto a quelle giapponese e tedesca, debba tenere in conto un certo grado di perdita di significato, che può tuttavia essere compensato da altrettante innovazioni o sperimentazioni nella lingua di arrivo. La negazione dell'esistenza di un originale, nella poetica di Tawada, può infatti essere vista come una rielaborazione del concetto stesso di 'traduzione' ed 'estraneità', che a sua volta permette di giocare con le possibilità che il plurilinguismo offre anche in traduzione, così da creare nuovi testi che non siano estranei o minoritari rispetto all'originale, ma complementari ad esso.

\section{Bibliografia}

Agnese, B., Ivanovic, C., Vlasta, S. (2015): Die Lücke im Sinn. Vergleichende Studien zu Yoko Tawada. Tübingen: Stauffenburg Verlag.

Benjamin, W. (1972): Die Aufgabe des Übersetzers. In: Gesammelte Schriften, Bd. IV/1. Frankfurt am Main: Suhrkamp.

Braidotti, R. (1995): Soggetto nomade. Femminismo e crisi della modernità. Roma: Donzelli [Trad. it di T. D’Agostini].

Comberiati, D. (2010): La letteratura postcoloniale italiana: definizioni, problemi, mappatura. In: Quarquarelli, L. (a cura di), Certi confini. Sulla letteratura italiana della migrazione, pp. 151-178. Milano: Morellini.

Gnisci, A. (2003): Creolizzare l'Europa: letteratura e migrazione. Roma: Meltemi.

Kloepfer, A. (1998): Also es gibt kein Original. Japanische Germanisten im Gespräch mit der Schriftstellerin Yoko Tawada. OAG-Notizen 11, pp. 11-19.

Kloepfer, A., Matsunaga, M. (2000): Yoko Tawada. Kritisches Lexikon der Gegenwartsliteratur, 64, pp. 2-17.

Kremnitz, G. (2004): Mehrsprachigkeit in der Literatur. Wie Autoren ihre Sprachen wählen. Wien: Praesens Verlag.

Lamping, D. (1992): Die literarische Übersetzung als dezentrale Struktur: Das Paradigma der Selbstübersetzung. In: Kittel, H. (a cura di), Geschichte, System, literarische Übersetzung Übersetzung, Bd. 5, pp. 212-227. Berlin: E. Schmidt.

Lusetti, C. (2017): Provare a ridirsi: l'autoriduzione come tappa di un processo migratorio in Amara Lakhous. Tricontre. Teoria Testo Traduzione, VII, pp. 109-127.

Moretto, I. (2010): I nuovi orizzonti della letteratura della migrazione: scrittori italiani in Germania e scrittori stranieri in Italia. Lingua Nostra, e Oltre - Numero monografico "Letteratura migrante", 3, pp. 72-80.

Moro, D. (2018a): La condizione dello 'straniero' tra ricerca e rifiuto dell'identità. In: Tawada, Y., Persona, pp. 9-34. Venezia: Libreria Editrice Cafoscarina. 
Moro, D. (2018b): Nota sull'autrice. In: Tawada, Y., Persona, pp. 98-108. Venezia: Libreria Editrice Cafoscarina.

Sgambati, G. (2012): Dalla Linguamadre alla Madrelingua, Yoko Tawada. Lettera Internazionale, 111 , pp. 45-46.

Sgambati, G. (2014): 'Es gibt kein Original!'. Mögliche italienische Übersetzungen des hybriden Schreibens von Yoko Tawada. Zibaldone - Zeitschrift für italienische Kultur der Gegenwart, 58, pp. 71-82.

Tawada, Y. (1989): Das Bad. Tübingen: Konkursbuch Verlag Claudia Gehrke. [Trad. de di P. Pörtner].

Tawada, Y. (1996): Talisman. Tübingen: Konkursbuch Verlag Claudia Gehrke.

Tawada, Y. (2003): Il bagno. Salerno: Edizioni Ripostes. [Trad. it di L. Aversa e L. Perrone Capano].

Tawada, Y. (2004): Schreiben im Netz der Sprachen. In: Kutzmutz, O., Waterhouse, P. (Hg.), Halbesachen. Dokument der Wolfenbütteler Übersetzergespräche, I-III, pp. 36-43.

Tawada, Y. (2011): Yuki no renshusei. Tokyo: Shinchosha.

Tawada, Y. (2013): Etüden im Schnee. Tübingen: Konkursbuch Verlag Claudia Gehrke. Tawada, Y. (2016): Memoirs of a polar bear. New York: New Directions [Trad. en di S. Bernofsky].

Tawada, Y. (2017): Memorie di un'orsa polare. Milano: Ugo Guarda Editore. [Trad. it di A. Iadicicco].

Tawada, Y. (2018): Persona. Venezia: Libreria Editrice Cafoscarina [Trad. it di D. Moro]. Wright, C. (2008): 'Writing in the 'Grey Zone': Exophonic Literature in Contemporary Germany. German as a Foreign Language Journal, 3, pp. 26-42.

\section{Sitografia}

Capano Perrone, L. (2008): Nuove migrazioni, http://www.exilderfrauen.it/nuove migrazioni_dettaglio.php?id=109 (accesso: 29.06.2019).

The Warwick Prize for Women in Translation, https://warwick.ac.uk/fac/cross_fac/ womenintranslation/winner2017/ (accesso: 29.06.2019). 